\title{
Myxoid liposarcoma with cartilaginous differentiation showing DDIT3 rearrangement
}

\author{
KAYO SUZUKI $^{1}$, TAKETOSHI YASUDA ${ }^{1}$, KENTA WATANABE $^{1}$, TAKESHI HORI ${ }^{2}$, \\ MASAHIKO KANAMORI $^{3}$ and TOMOATSU KIMURA ${ }^{1}$ \\ ${ }^{1}$ Department of Orthopaedic Surgery, University of Toyama, Toyama, Toyama 930-0194;
${ }^{2}$ Department of Orthopaedic Surgery, Iiyama Red Cross Hospital, Iiyama, Nagano 389-2295;
${ }^{3}$ Department of Human Science 1, University of Toyama, Toyama, Toyama 930-0194, Japan
}

Received December 23, 2015; Accepted February 23, 2017

DOI: 10.3892/ol.2017.7056

\begin{abstract}
Myxoid liposarcoma (MLPS) is the second most common histologic subtype of liposarcoma. However, cartilaginous differentiation within MLPS is an extremely rare phenomenon, with only 7 cases of MLPS with cartilaginous differentiation reported to date. The majority of MLPS cases show the $\mathrm{t}(12 ; 16)(\mathrm{q} 13 ; \mathrm{p} 11)$ translocation, resulting in the fused in sarcoma-DNA damage-inducible transcript 3 (FUS-DDIT3) fusion gene. This fusion gene as a hallmark of MLPS is very useful for differential diagnosis from other soft tissue sarcomas, and the associated protein, FUS-DDIT3, performs an important role in the phenotypic selection of targeted multipotent mesenchymal cells during oncogenesis. In this report, a case of MLPS with cartilaginous differentiation that occurred in the thigh of a 44-year-old woman is described. Histopathologically, the tumor was composed of a typical myxoid liposarcoma area and a mature hyaline cartilaginous area. Using fluorescence in situ hybridization analysis, rearrangement of the DDIT3 gene was detected in not only the liposarcomatous area but also in the chondrocytes of the cartilaginous area. Based on these findings, the cartilaginous differentiation area appears to be partially associated with oncogenesis through the specific fusion gene FUS-DDIT3.
\end{abstract}

Correspondence to: Dr Taketoshi Yasuda, Department of Orthopaedic Surgery, University of Toyama, 2630 Sugitani, Toyama, Toyama 930-0194, Japan

E-mail: yasuda@med.u-toyama.ac.jp

Abbreviations: MLPS, myxoid liposarcoma; ALT, atypical lipomatous tumor; DLPS, dedifferentiated liposarcoma; PLPS, pleomorphic liposarcoma; FUS, fused in liposarcoma; DDIT3, DNA damage-inducible transcript 3; EWSR1, Ewing's sarcoma; FISH, fluorescence in situ hybridization; MPCs, mesenchymal progenitor cells; Opn, osteopontin; Col11a2, alpha 2 chain of type XI collagen; PPAR- $\gamma$, peroxisome proliferator activated receptor- $\gamma$

Key words: MLPS, cartilaginous differentiation, cytogenetics, DDIT3 rearrangement

\section{Introduction}

Liposarcoma is one of the most common soft tissue sarcomas in adults, representing an estimated $17-25 \%$ of all sarcomas $(1,2)$. The tumor occurs at all ages, but is most commonly identified in individuals between 40 and 60 years of age. A total of four distinct histologic subtypes of liposarcoma are recognized by the World Health Organization: i) Atypical lipomatous tumor (ALT); ii) dedifferentiated liposarcoma (DLPS); iii) myxoid liposarcoma (MLPS); and iv) pleomorphic liposarcoma (PLPS). ALT and DLPS are genetically defined by a giant marker or ring chromosomes with an amplification on chromosome 12 affecting, among others, the genes mouse double minute 2 homolog and cyclin-dependent kinase 4 (3). MLPS denotes one such entity and is the second most common liposarcoma following ALT (4). A significant proportion of MLPS cases have the major cytogenetic hallmark of the $t(12 ; 16)$ (q13;p11) chromosomal translocation. This translocation leads to fusion of fused in liposarcoma (FUS; also termed translocated in liposarcoma) and DNA damage-inducible transcript 3 (DDIT3; also termed CCAAT/enhancer-binding protein homologous protein) genes, resulting in the production of the FUS-DDIT3 fusion protein (5). In another subset of MLPS, a minor chromosomal translocation, $\mathrm{t}(12 ; 22)(\mathrm{q} 13 ; \mathrm{q} 12)$, results in fusion of the Ewing's sarcoma (EWSR1) and DDIT3 genes (6). PLPS is less frequent and harbors a complex genomic profile with numerous gains and losses similar to the genomic profile observed in poorly differentiated sarcoma (7).

The histopathological findings of MLPS are typically composed of well-circumscribed lobulated tumors. These contain a mixture of uniform round to oval-shaped nonlipogenic mesenchymal cells and signet ring lipoblasts in a prominent myxoid background, rich in a delicate arborizing capillary vasculature (4). Although it is an extremely rare phenomenon, 7 cases of MLPS with cartilaginous differentiation have been reported to date (8-12). When this heterogeneous component is present in MLPS, it may be difficult to distinguish MLPS from malignant mesenchymoma or chondroid lipoma on histopathological examination. Detection of the FUS-DDIT3 fusion gene is useful for the differential diagnosis of these tumors. However, only $3 / 7$ of previous cases showed the FUS-DDIT3 fusion gene in the 
typical liposarcomatous and cartilaginous components on cytogenetic analysis (10-12). As there are few MLPS cases with this heterogeneous component, whether the cartilaginous differentiation in MLPS affects the clinical features and prognosis has not been determined.

In the present study, an additional case of MLPS with cartilaginous differentiation in which the FUS-DDIT3 fusion gene was detected by fluorescence in situ hybridization (FISH) analysis is described, and the literature on MLPS with cartilaginous differentiation is reviewed. The purpose of this study was to clarify the clinical characteristics in MLPS with cartilaginous differentiation.

\section{Materials and methods}

Patient characteristics. A 44-year-old woman had noted a painless mass in the left thigh. The mass had been present for 4 years and had slowly increased in size. The mass was elastic and soft, with no signs of inflammation, but its borders were unclear. Radiological studies showed an expansion of the soft tissue, but no bony changes or calcifications. Magnetic resonance imaging revealed a $21 \mathrm{~cm}$ mass spreading on the lateral muscle component on the axial aspect of the left thigh. The lesion appeared isointense to skeletal muscle on T1-weighted images and heterogeneously hyperintense and partially hypointense on T2-weighted images (Fig. 1). A needle biopsy was performed, and a histologic diagnosis of MLPS was made based on the uniform round to oval-shaped nonlipogenic mesenchymal cells and a number of small lipoblasts within the myxoid stroma. The mass was excised with wide margins, and a sterile portion was submitted for cytogenetic analysis. The patient has had no recurrence or metastasis for 12 months subsequent to the surgery. The present report was approved by the Ethics Committee of the Toyama University Hospital (Toyama, Japan). The patient provided written consent for the report.

Immunohistochemical analysis. MIB-1 labeling index was evaluated by immunohistochemical staining using Ki-67 antibody (1:100; catalog no. M7240; Dako; Agilent Technologies, Inc., Santa Clara, CA, USA). Formalin-fixed paraffin-embedded tissues were cut into $4 \mu \mathrm{m}$ sections and mounted onto coated slides. Following deparaffinization and rehydration, antigen retrieval was carried out by heating with a microwave for $10 \mathrm{~min}$ in a citrate buffer solution $(10 \mathrm{mM})$ at $\mathrm{pH}$ 6.0. Following $30 \mathrm{~min}$ blocking with $5 \%$ bovine serum albumin (Sigma-Aldrich, St. Louis, MO, USA) at room temperature, the slide was incubated for $1 \mathrm{~h}$ at room temperature with the Ki-67 antibody. Next, the slide section was incubated with the biotinylated secondary antibody (polyclonal goat biotinylated anti-mouse immunoglobulin; catalog no. E0433; 1:200; Dako, Agilent Technologies) for $30 \mathrm{~min}$ at room temperature and subsequently incubated with the avidin-biotin-peroxidase complex (Vector Laboratories, Burlingame, CA, USA) for $30 \mathrm{~min}$. Finally, staining was visualized with 3,3'-diamino-benzidine-tetra hydrochloride. The slide was counterstained with Mayer's hematoxylin. The MIB-1 labeling index was expressed as the percentage of positive cells among 100 tumor cells in an area with high cell density.
Cytogenetic analysis. Representative fresh tissue from the surgical resection sample was received for conventional cytogenetic analysis. Culturing, harvesting, and preparation of slides were performed as previously described (13). Briefly, the tissues were disassociated mechanically and enzymatically and cultured at $37^{\circ} \mathrm{C}$ in RPMI 1640 (Sigma-Aldrich; Merck KGaA, Darmstadt, Germany) supplemented with $20 \%$ fetal bovine serum (ICN Biomedicals, Inc., Aurora, OH, USA) for 3-8 days. Cultured cells received overnight exposure to colcemid $(0.02 \mu \mathrm{g} / \mathrm{ml})$. Following hypotonic treatment $(0.8 \%$ sodium citrate for $20 \mathrm{~min}$ at room temperature), the cells were fixed three times with methanol:glacial acetic acid (3:1) at room temperature. Chromosome analysis was performed on G-band by trypsin and Giemsa (GTG) banding. GTG banding was performed by incubating the glass slides in a $0.05 \%$ trypsin solution at $37^{\circ} \mathrm{C}$ for $15 \mathrm{sec}$, followed by rinsing the slides in phosphate-buffered saline buffer and staining in a $5 \%$ Giemsa stain for $8 \mathrm{~min}$. The slides were rinsed with water and air-dried. The karyotypes were expressed according to the International System for Human Cytogenetic Nomenclature 2013 (14).

FISH analysis. An interphase FISH analysis was performed on formalin-fixed, paraffin-embedded tissue sections of the tumor using a commercially available DDIT3 (12q13) dual-color, break-apart rearrangement probe (catalog no. 5J48-05; Abbott Molecular Inc., Des Plaines, IL, USA). The probe consists of a mixture of two FISH DNA probes. The first probe is a $700 \mathrm{~kb}$ probe (orange), which flanks the 3' side of the DDIT3 gene (12q13). The second one is a $2663 \mathrm{~kb}$ probe (green), which flanks the 5' side of the DDIT3 gene (12q13). The FISH analysis was performed according to the manufacturer's protocol using Vysis Paraffin Pretreatment IV and Post-Hybridization Wash buffer kit (Abbott Molecular Inc.), and as described in a previous study (15). Hybridization signals were visualized with an epi-fluorescence microscope, and images were captured on a charge-coupled-device camera. A total of 50 nuclei showing both green and orange signals were counted, and the percentage of the fused signals in 50 nuclei was calculated by two different observers. The fusion signal appeared yellow as orange and green signals overlap partially or totally with oil immersed objective lens. In cases where the nuclei overlapped and the complete area of each nucleus was not visible, or the nuclei were too close together to determine boundaries, the signals were not counted.

\section{Results}

Histology. Grossly, the specimen measured $21 \mathrm{x} 8 \mathrm{x} 4 \mathrm{~cm}$, and the tumor had multinodular areas and a bluish cartilaginous spot (indicated by the arrows in Fig. 2). On low power microscopy, a liposarcomatous area was adjacent to the cartilaginous area (Fig. 3A). The lesion of the liposarcomatous component showed typical myxoid liposarcoma, composed of the proliferation of spindle to oval-shaped nonlipogenic cells and small lipoblasts in myxoid stroma and a plexiform capillary vascular network. The tumor cell was atypical with a high nuclear-cytoplasmic ratio and dense chromatin. Additionally, the size and of the nuclei were moderately or slightly enlarged, and the shape of the nuclei were irregular compared with 


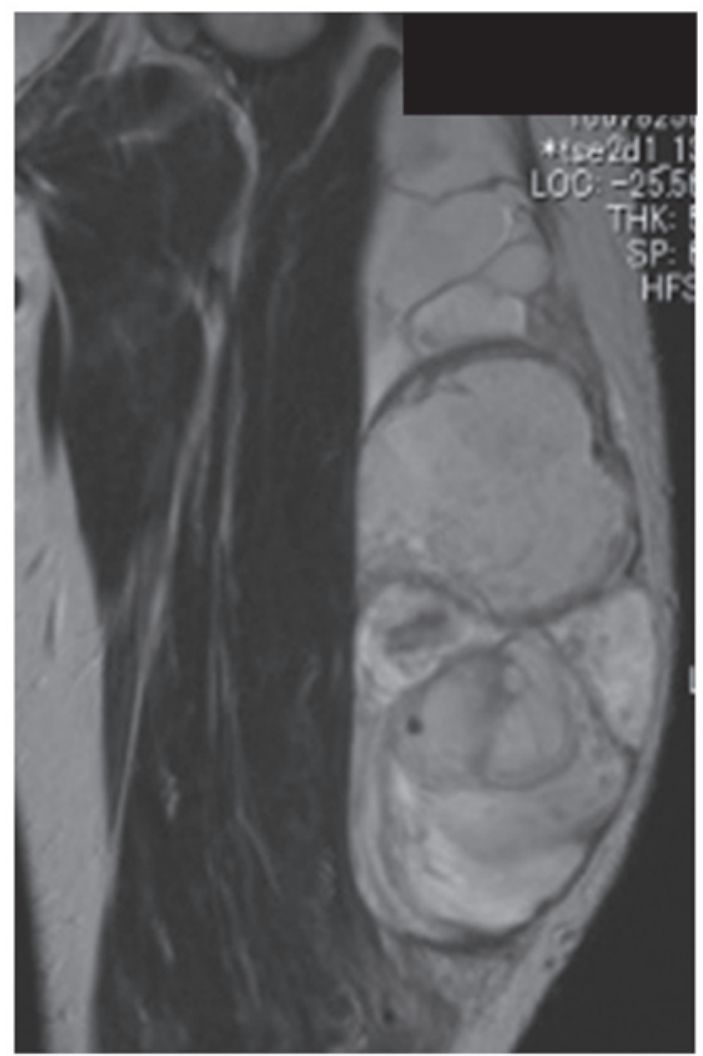

Figure 1. T2-weighted magnetic resonance images of the left thigh. A coronal-plane image shows the mass occurring in the vastus lateralis and a heterogeneous pattern with low and high signal intensities.

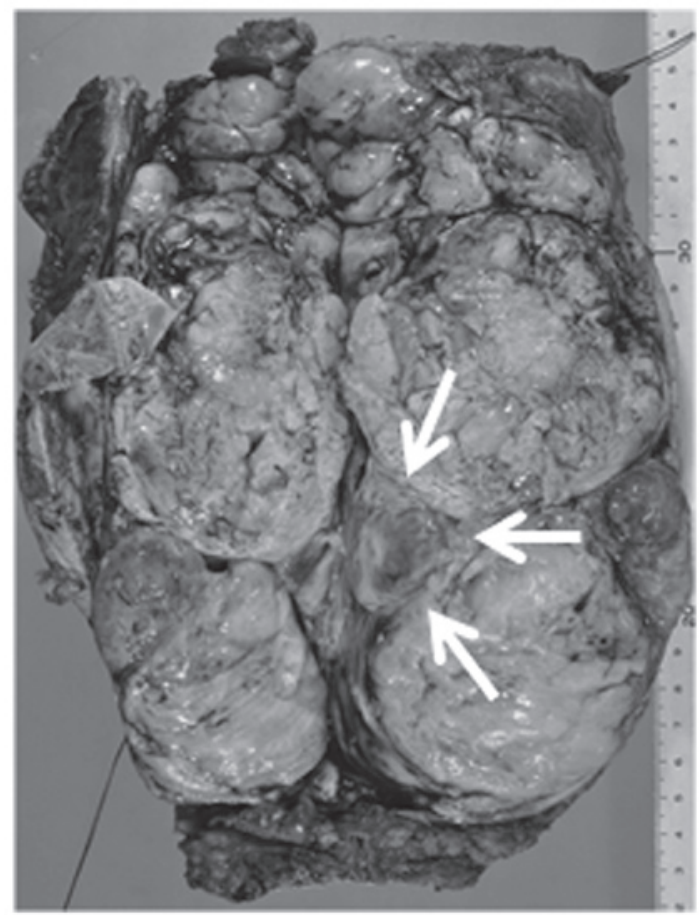

Figure 2. Gross appearance. The mass was $21 \times 8 \times 4 \mathrm{~cm}$, and in the sectioned surface of the coronal plane, the tumor has multinodular areas and a bluish cartilaginous spot (arrow).

normal cells (Fig. 3B). Significant mitotic figures were rare. It was found that the MIB-1 labeling index was $\sim 1 \%$. Small
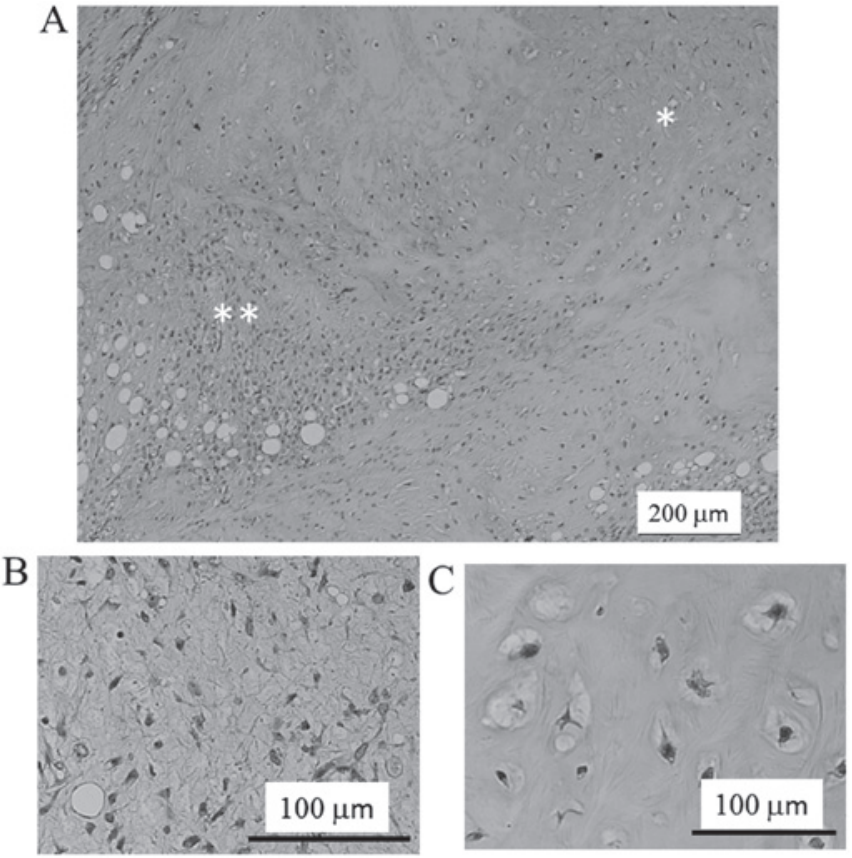

Figure 3.Histopathological findings of the resected tumor.(A) Microscopically, a liposarcomatous component $\left(^{* *}\right)$ and a cartilaginous component $\left(^{*}\right)$ are adjacent (hematoxylin and eosin stain; magnification, $x 40$ ). (B) The liposarcomatous component exhibits proliferation of atypical spindle-shaped round cells and the presence of myxoid stroma (hematoxylin and eosin stain; magnification, x100). (C) The cartilaginous component demonstrated mature hyaline cartilage (hematoxylin and eosin stain; magnification, x100).

areas ( $20 \%)$ of the tumor had a cartilaginous component with mild cellularity of mature chondrocytes and focally atypical chondrocytes (Fig. 3C). Based on these findings, the diagnosis of MLPS with cartilaginous differentiation was made.

Cytogenetic analysis. In total, 18/20 analyzed metaphase cells were karyotypically abnormal and characterized by the following chromosomal complement: 46, XX, add (1) (p13), -2 , der (4) $\mathrm{t}(1 ; 4)(\mathrm{q} 11 ; \mathrm{p} 16)$, del (7) (p11.2), der (12) ?t(12;16) (q13;p11.2), -14, -16, der (16) t(7;16)(p11.2;p11.2), -17, add (17) (q25), add (21) (p11.2), +mar1, +mar2, +mar3, +mar4 (Fig. 4).

FISH. An interphase FISH analysis showed that $>10 \%$ of the cells from the typical MLPS area and the chondrocytes of the cartilaginous component showed a split signal pattern of one green and one orange, demonstrating a rearrangement in the DDIT3 gene (Fig. 5A and B).

\section{Discussion}

MLPS is the second most common histologic subtype following ALT in liposarcoma and accounts for $15-20 \%$ of all liposarcomas (4). MLPS usually occurs in deep soft tissues of the extremities, particularly the thigh. MLPS is histopathologically characterized by the mixture of uniform round to oval-shaped non-lipogenic cells and small signet ring lipoblasts in a prominent myxoid stroma. MLPS with cartilaginous differentiation is extremely rare; to the best of our knowledge, there have been only 7 previously reported cases (8-12). The associations between clinical features and 
Table I. Characteristics of MLPS with cartilaginous differentiation in previous studies.

Detection of FUS-DDIT3

\begin{tabular}{|c|c|c|c|c|c|c|c|c|c|c|c|}
\hline Case & Age/sex & Site & $\begin{array}{c}\text { Duration, } \\
\text { months }\end{array}$ & $\begin{array}{c}\text { Size, } \\
\mathrm{cm}\end{array}$ & Analysis & $\begin{array}{l}\text { Liposarco- } \\
\text { matous area }\end{array}$ & $\begin{array}{c}\text { Cartilaginous } \\
\text { area }\end{array}$ & $\begin{array}{l}\text { MIB-1 } \\
\text { LI, \% }\end{array}$ & Therapy & $\begin{array}{r}\text { Prognosis } \\
\text { (months) }\end{array}$ & (Refs.) \\
\hline 1 & $37 / \mathrm{M}$ & Thigh & 11 & 13 & NA & NA & NA & NA & CR, RT & NED (45) & (8) \\
\hline 2 & $42 / \mathrm{M}$ & Thigh & 7 & 9 & NA & NA & NA & NA & CR, RT & NED (37) & (8) \\
\hline 3 & $63 / \mathrm{M}$ & Thigh & 2 & 8 & NA & NA & NA & NA & CR, RT & NED (36) & (8) \\
\hline 4 & $26 / M$ & Thigh & NA & 11 & $\mathrm{G}$ band & NA & NA & NA & CR, RT & NED (36) & (9) \\
\hline 5 & $47 / \mathrm{M}$ & Thigh & 12 & 13 & RT-PCR & + & + & NA & $\mathrm{CR}$ & NED (18) & (10) \\
\hline 6 & $45 / \mathrm{F}$ & Thigh & 3 & 11 & RT-PCR & + & + & $<5$ & CR, RT & NED (6) & (11) \\
\hline 7 & $37 / \mathrm{M}$ & Thigh & 12 & 8 & FISH & + & + & NA & CR, RT & NED (6) & (12) \\
\hline Present & $44 / \mathrm{F}$ & Thigh & 48 & 21 & FISH & + & + & 1 & $\mathrm{CR}$ & NED (12) & \\
\hline
\end{tabular}

FUS-DDIT3, fused in sarcoma-DNA damage-inducible transcript; duration, from presence of symptom to initial diagnosis; MIB-1 LI, MIB-1 labeling index; M, male; F, female; G band, Giemsa banding; RT-PCR, reverse transcription polymerase chain reaction; FISH, fluorescence in situ hybridization; NA, not available; CR, complete resection; RT, radiation therapy; NED, no evidence of disease; MLPS, myxoid liposarcoma.

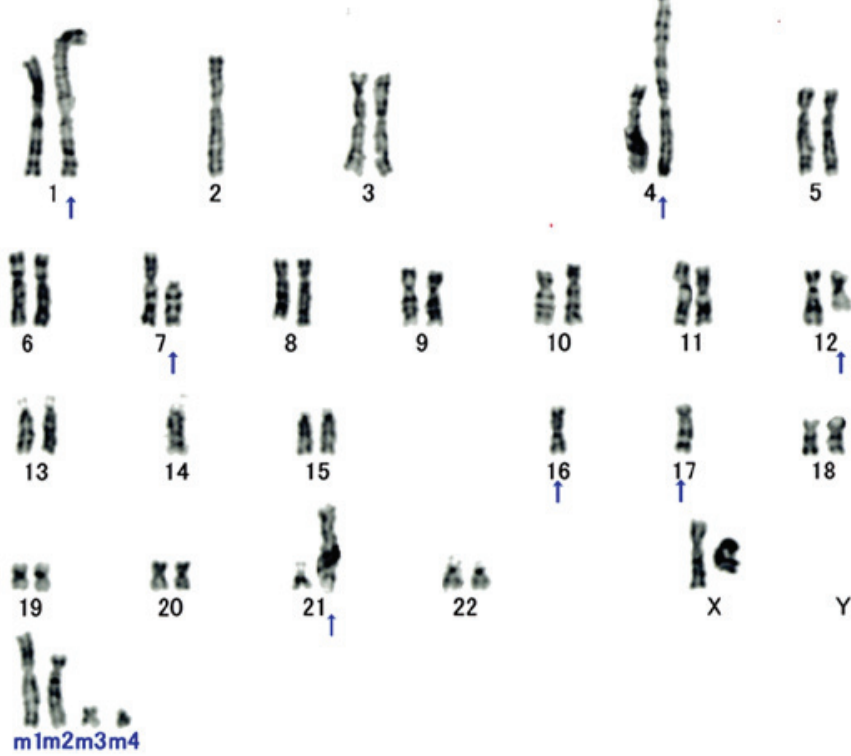

Figure 4. Cytogenetic analysis. Karyotyping of patient surgical specimen shows 46, XX, add(1)(p13), -2, der(4) t(1;4)(q11;p16), del(7) (p11.2), der(12) ?t(12;16)(q13;p11.2), -14, -16, der (16)t(7;16)(p11.2;p11.2), -17, add(17) (q25), add (21)(p11.2), +mar1, +mar2, +mar3, +mar4. All aberrant chromosomes are indicated with arrows. The translocation of $\mathrm{t}(12 ; 16)(\mathrm{q} 13 ; \mathrm{p} 11.2)$ generates a FUS-DDIT3 fusion transcript and is a cytogenetic hallmark of myxoid liposarcoma.

the cartilaginous differentiation in MLPS are unclear, and the seven previous cases and the present case are listed in Table I. The range of ages was 26-63 years (median, 43 years), and the site of involvement was the thigh in all cases, corresponding to the most common site in MLPS (4). The median size of the tumors was $11 \mathrm{~cm}$ (range, $8-21 \mathrm{~cm}$ ). Nishida et al (16) reported that MLPS occurred in the extremities and trunk wall with a tumor size of $<10 \mathrm{~cm}$ in $64 \%$ of patients, while $36 \%$ exhibited a tumor size of $\geq 10 \mathrm{~cm}$. In the previous largest study on MLPS, a tumor size of $>10 \mathrm{~cm}$ was demonstrated to be an

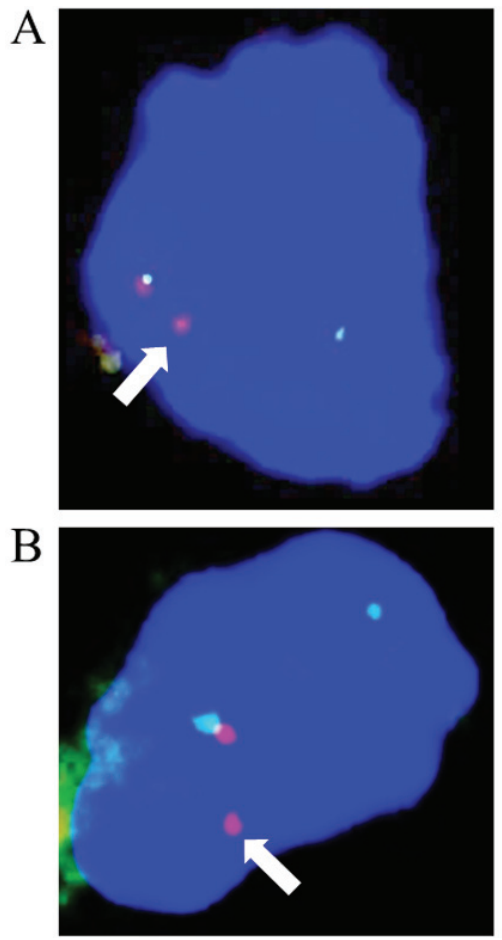

Figure 5. Fluorescent in situ hybridization analysis. The DDIT3 (12q13) breakpoint flanking probe set (proximal portion, orange; distal portion, green) shows splitting of the orange and green signals, indicating disruption of DDIT3. (A) Tumor cells in the liposarcomatous area. (B) Tumor cells in the cartilaginous area. Arrows indicate orange signals. DDIT3, DNA damage-inducible transcript 3 .

independent prognostic factor for disease-specific survival and metastasis-free survival (17). However, all 7 cases of MLPS with cartilaginous differentiation had a good prognosis, with no evidence of disease at final follow-up. Furthermore, analysis of the MIB-1 labeling index, a significant prognostic factor for overall survival in MLPS (18), demonstrated low values in $2 / 7$ measurable cases $(<5$ and $1 \%$ ). From the 
finding of a low MIB-1 index, it is thought that MLPS with cartilaginous differentiation has low malignancy and a good prognosis. These findings indicate that the presence of cartilaginous differentiation within MLPS may be a good prognostic factor.

As MLPS with cartilaginous differentiation is a rare condition, the differential diagnosis from other benign or malignant soft tissue sarcomas, such as chondroid lipoma, extraskeletal chondroma, extraskeletal myxoid chondrosarcoma and malignant mesenchymoma, is difficult. Although chondroid lipoma and extraskeletal chondroma have cells with a similar appearance to lipoblasts (6), the presence of a plexiform capillary vascular network, is a characteristic of MLPS. Myxoid chondrosarcomas are different in that they show chondrocyte atypia (19). The cartilaginous area within MLPS is composed of mature hyaline cartilage without chondrocyte atypia. Malignant mesenchymoma is defined as a malignant soft tissue tumor that consists of two or more distinctly different mesenchymal components in addition to fibrosarcomatous elements (20). In the differential diagnosis among these benign and malignant soft tissue tumors, the detection of the fusion gene specific to MLPS, FUS-DDIT3 or EWSR1-DDIT3, is extremely useful.

The FUS-DDIT3 fusion protein was reported to be associated with the oncogenesis of MLPS by Riggi et al (21). The expression of FUS-DDIT3 fusion protein in primary mesenchymal progenitor cells (MPCs) induced the development of MLPS-like tumors in mice (21). The expression of FUS-DDIT3 may be the initiating event in MLPS pathogenesis, and MPCs may constitute one cell type from which MLPS originates. In addition, microarray-based analysis of FUS-DDIT3-transformed adipose-derived mesenchymal stem cells revealed downregulation of osteopontin (Opn) and the alpha 2 chain of type XI collagen (Col11a2) mRNA levels, with preservation of peroxisome proliferator activated receptor- $\gamma$ (PPAR- $\gamma$ ) gene expression (22). We have previously studied the EWSR1-DDIT3 mediated phenotypic selection of putative target multipotent mesenchymal cells during MLPS development through Opn and Col11a2 downregulation (23). These fusion genes as a hallmark of MLPS are very useful for differential diagnosis from other soft tissue sarcomas, and the associated protein FUS-DDIT3 or EWSR1-DDIT3 performs an important role in the phenotypic selection of targeted multipotent mesenchymal cells during MLPS development. The rearrangement of the DDIT3 gene was shown by FISH analysis, not only in the typical liposarcomatous area, but also in the cartilaginous area. FUS-DDIT3 may participate in the process producing cartilage differentiation. To date, there have been only 4 cases including the present case in which the hallmark fusion gene was detected in lipomatous and cartilaginous components of MLPS, as summarized in Table I. The mechanism of cartilaginous differentiation induction within MLPS remains unclear. Previous studies (21-23) indicated that chondroid differentiation from mesenchymal progenitor cells may be suppressed by fusion protein through oncogenesis in typical MLPS. However, though FUS-DDIT3 is detected in both the liposarcomatous and cartilaginous areas in MLPS with cartilaginous differentiation, this tumor shows clinical and pathological differences in prognosis and proliferation activity from typical MLPS. Based on these findings, in addition to the function of FUS-DDIT3 fusion gene, another molecular mechanism or a modified mechanism in the production of cartilaginous differentiation may be present. The mechanism of cartilage differentiation within MLPS and its clinical significance will become clear in the future with examination of additional cases.

There were 3 limitations in the present study. First, the number of MLPS cases with cartilaginous differentiation was small, only 8 cases including the present case. The second limitation was that there were only two cases in which the MIB-1 labeling index was reported; this is one of the important malignant pathological features. Finally, in $4 / 8$ cases the follow-up time from the operation was $<2$ years. From the viewpoint of these limitations, additional cases and long-term follow-up are necessary to clarify whether the prognosis of MLPS with cartilaginous differentiation is better compared with that of typical MLPS.

In conclusion, a rare case of MLPS with cartilaginous differentiation was described, and DDIT3 rearrangement was demonstrated by FISH analysis in both lipomatous and cartilaginous components. The mechanism of cartilaginous differentiation in MLPS may be partially associated with the FUS-DDIT3 fusion gene.

\section{Acknowledgements}

The present study was supported in part by the Grants in Aid for Scientific Research (Japan Society for the Promotion of Science; grant no. 24592227). The authors are grateful to Professor Joji Imura, Department of Pathology, University of Toyama (Toyama, Japan), for discussion on histopathological diagnosis.

\section{References}

1. Weiss SW and Goldblum JR: Liposarcoma. In: Enzinger and Weiss's Soft Tissue Tumors, 5th edition. Enzinger Weiss SW and Goldblum JR (Eds), Mosby Inc., St. Louis: pp477-516, 2008.

2. Dei Tos AP and Pedeutour F: Atypical lipomatous tumor. In: WHO Classification of Tumours of Soft Tissue and Bone. Fletcher CDM, Bridge JA, Hogendoorn PCW and Mertens F (Eds), IARC, Lyon: pp33-43, 2013.

3. Coindre JM, Pédeutour F and Aurias A: Well-differentiated and dedifferentiated liposarcomas. Virchows Arch 456: 167-179, 2010.

4. Antonescu CR and Ladanyi M: Myxoid liposarcoma. In: WHO Classification of Tumours of Soft Tissue and Bone. Fletcher CDM, Bridge JA, Hogendoorn PCW and Mertens F (Eds), IARC, Lyon: pp39-41, 2013.

5. Antonescu CR, Tschernyavsky SJ, Decuseara R, Leung DH, Woodruff JM, Brennan MF, Bridge JA, Neff JR, Goldblum JR and Ladanyi M: Prognostic impact of P53 status, TLS-CHOP fusion transcript structure, and histological grade in myxoid liposarcoma: A molecular and clinicopathologic study of 82 cases. Clin Cancer Res 7: 3977-3987, 2001.

6. Hosaka T, Nakashima Y, Kusuzaki K, Murata H, Nakayama T, Nakamata T, Aoyama T, Okamoto T, Nishijo K, Araki N, et al: A novel type of EWS-CHOP fusion gene in two cases of myxoid liposarcoma. J Mol Diagn 4: 164-171, 2002.

7. Idbaih A, Coindre JM, Derré J, Mariani O, Terrier P, Ranchère D, Mairal A and Aurias A: Myxoid malignant fibrous histiocytoma and pleomorphic liposarcoma share very similar genomic imbalances. Lab Invest 85: 176-181, 2005.

8. Siebert JD, Williams RP and Pulitzer DR: Myxoid liposarcoma with cartilaginous differentiation. Mod Pathol 9: 249-252, 1996.

9. Dijkhuizen T, Molenaar WM, Hoekstra HJ, Wiersema J and van den Berg E: Cytogenetic analysis of a case of myxoid liposarcoma with cartilaginous differentiation. Cancer Genet Cytogenet 92: $141-143,1996$. 
10. Wei YC, Li CF, Eng HL, Yeh MC, Lin CN and Huang HY: Myxoid liposarcoma with cartilaginous differentiation: Identification of the same type II TLS-CHOP fusion gene transcript in both lipogenic and chondroid components. Appl Immunohistochem Mol Morphol 15: 477-480, 2007.

11. Kim H, Hwangbo W, Ahn S, Kim S, Kim I and Kim CH: Myxoid liposarcoma with cartilaginous differentiation: A case study with cytogenetical analysis. Korean J Pathol 47: 284-288, 2013.

12. Ioannou MG, Kouvaras E, Papamichali R, Karachalios T and Koukoulis G: Myxoid liposarcoma with cartilaginous differentiation: A case study with fish analysis and review of the literature. Pathol Res Pract 209: 666-669, 2013.

13. Nishio J, Althof PA, Bailey JM, Zhou M, Neff JR, Barr FG, Parham DM, Teot L, Qualman SJ and Bridge JA: Use of a novel FISH assay on paraffin-embedded tissues as an adjunct to diagnosis of alveolar rhabdomyosarcoma. Lab Invest 86: 547-556, 2006.

14. Simons A, Shaffer LG and Hastings RJ: Cytogenetic nomenclature: Changes in the ISCN 2013 compared to the 2009 edition. Cytogenet Genome Res 141: 1-6, 2013.

15. Yamaguchi U, Hasegawa T, Morimoto Y, Tateishi U, Endo M, Nakatani F, Kawai A, Chuman H, Beppu Y, Endo M, et al: A practical approach to the clinical diagnosis of Ewing's sarcoma/primitive neuroectodermal tumor and other small round cell tumors sharing EWS rearrangement using new fluorescence in situ hybridization probes for EWSR1 on formalin fixed, paraffin wax embedded tissue. J Clin Pathol 58: 1051-1056, 2005.

16. Nishida $\mathrm{Y}$, Tsukushi $\mathrm{S}$, Nakashima $\mathrm{H}$ and Ishiguro $\mathrm{N}$ : Clinicopathologic prognostic factors of pure myxoid liposarcoma of the extremities and trunk wall. Clin Orthop Relat Res 468: 3041-3046, 2010.
17. Fiore M, Grosso F, Lo Vullo S, Pennacchioli E, Stacchiotti S, Ferrari A, Collini P, Lozza L, Mariani L, Casali PG and Gronchi A: Myxoid/round cell and pleomorphic liposarcomas: Prognostic factors and survival in a series of patients treated at a single institution. Cancer 109: 2522-2531, 2007.

18. Tateishi U, Hasegawa T, Beppu Y, Kawai A and Moriyama N: Prognostic significance of grading (MIB-1 system) in patients with myxoid liposarcoma. J Clin Pathol 56: 579-582, 2003.

19. Enzinger FM and Shiraki M: Extraskeletal myxoid chondrosarcoma. An analysis of 34 cases. Hum Pathol 3: 421-435, 1972.

20. Weiss SW and Goldblum JR. Malignant mesenchymoma. In: Enzinger and Weiss's Soft tissue tumors, 5th edition. Enzinger Weiss SW and Goldblum JR (Eds), Mosby Inc., St. Louis: pp1213-1214, 2008.

21. Riggi N, Cironi L, Provero $\mathrm{P}$, Suvà ML, Stehle JC, Baumer K, Guillou L and Stamenkovic I: Expression of the FUS-CHOP fusion protein in primary mesenchymal progenitor cells gives rise to a model of myxoid liposarcoma. Cancer Res 66: 7016-7023, 2006.

22. Rodriguez R, Rubio R, Gutierrez-Aranda I, Melen GJ, Elosua C, García-Castro J and Menendez P: FUS-CHOP fusion protein expression coupled to p53 deficiency induces liposarcoma in mouse but not in human adipose-derived mesenchymal stem/stromal cells. Stem Cells 29: 179-192, 2011.

23. Suzuki K, Matsui Y, Higashimoto M, Kawaguchi Y, Seki S, Motomura H, Hori T, Yahara Y, Kanamori M and Kimura T: Myxoid liposarcoma-associated EWSR1-DDIT3 selectively represses osteoblastic and chondrocytic transcription in multipotent mesenchymal cells. PLoS One 7: e36682, 2012. 